\title{
Nonlinear Channels: Predistortion or Enhanced Detection?
}

\author{
Armando Vannucci and Riccardo Raheli \\ Dipartimento di Ingegneria dell'Informazione \\ Università di Parma, I - 43100 Parma, Italy
}

\begin{abstract}
Using a union bound on the error probability of optimal receivers for nonlinear channels, this work points out important factors affecting the error performance in the simple case of $M$-ary Pulse Amplitude Modulation ( $M$-PAM) with no intersymbol interference and unlimited transmission bandwidth. Performance is compared to that obtainable when the nonlinear device is perfectly linearized under a peak power constraint. Considered nonlinearities include third-order polynomials and soft-limiters. Simulation results are in good agreement with the derived theoretical bounds. The analysis suggests that margins for improved performance exist, with respect to the performance obtainable by perfect linearization, when the cardinality of the signal set is not large (e.g., 4- or 8-PAM) and the nonlinearity is not severely saturated.
\end{abstract}

\section{INTRODUCTION}

$\mathrm{I}_{\mathrm{t} w a x}^{\mathrm{T}}$ $\mathrm{T}$ has been shown through a brute force approach that performance degradation in dispersive nonlinear channels can be almost completely recovered through appropriate receiver design [1]. Optimal receivers for such channels have been proposed by Mesiya, Mc Lane and Campbell [2], for Phase Shift Keying (PSK) modulation formats, and van Etten and van Vugt [3], for general linearly modulated signals. The first paper showed that the power loss with respect to an equal-power linear channel can be kept within about a dB, whereas the latter showed that in the case of binary signaling error performance undergoes an improvement when Inter-Symbol Interference (ISI) is allowed on the nonlinear channel.

The aim of the present work is to compare the error performance obtainable on nonlinear channels to that achievable on the linear channel which results from a perfect analog predistortion of the nonlinear device, typically a high power amplifier. In order to gain an in depth view of the factors affecting performance, we will limit our attention to the simple case in which the nonlinear device has no AM/PM distortion and the bandwidth of the physical channel is large enough to avoid intersymbol interference. Moreover, a monodimensional signaling scheme, namely multilevel PAM, will be taken into account.

First we will consider cubic nonlinearities; upper and lower bounds to the symbol error probability will be derived for the considered channel with various degrees of distortion. Since cubic characteristics do not

This work was performed partly within a research cooperation between the Dipartimento di Ingegneria dell'Informazione, Università di Parma, Italy and Italtel S.p.A., Milano, Italy.

0-7803-5106-1/98/\$10.00 @ 1998 IEEE cause large saturating effects on the transmitted signal, we will also consider a soft-limiting device, with various degrees of the Input Back-Off (IBO) relative to the saturating operation point. The derived bounds will be shown to be in good agreement with simulation results. The effects of constellation density and transmission filter response will also be addressed.

The simplifying assumptions made on the channel model could be relaxed by allowing ISI caused by a limited channel bandwidth; however, the in-depth speculations made in section III-B on the present case would no longer be feasible and one should resort to numerical calculations [4].

\section{SYSTEM MODEL AND OPTIMAL RECEIVER PERFORMANCE}

Fig. 1 sketches the baseband equivalent of a nonlinear system in which the information symbols $\left\{a_{n}\right\}$ generate, through a transmission filter with impulse response $p(t)$, a linearly modulated signal

$$
x(t)=\sum_{n} a_{n} p(t-n T)
$$

which is fed to a bandpass memoryless nonlinear amplifying device whose transfer characteristic entails $\mathrm{AM} / \mathrm{AM}$ and $\mathrm{AM} / \mathrm{PM}$ distortion. Denoting by $F[\cdot]$ and $\Phi[\cdot]$ these distortion characteristics, the baseband equivalent signal $y(t)$ at the output of the amplifier has expression

$$
y(t)=F[|x(t)|] \mathrm{e}^{j \Phi[|x(t)|]} \mathrm{e}^{j L x(t)} .
$$

This signal is further fed to a channel with impulse response $h(t)$ which outputs a signal that will be denoted by $z^{\prime}(\mathbf{a}, t)$ to underline its dependence on the symbol sequence a. Additive White Gaussian Noise (AWGN) $n(t)$ is added at the output of the channel. The receiver processes the observed signal in order to perform Maximum Likelihood (ML) detection of the transmitted sequence.

It is well known [5] that the probability of detecting a sequence $\hat{\mathbf{a}}$ different from the actual transmitted sequence a can be upper bounded by the pairwise error probability as*

$$
\begin{array}{r}
P(\hat{\mathbf{a}} \mid \mathbf{a}) \leq Q\left[\frac{d(\hat{\mathbf{a}}, \mathbf{a})}{\sqrt{2 N_{0}}}\right] \\
{ }^{*} Q(\boldsymbol{x}) \triangleq \frac{1}{\sqrt{2 \pi}} \int_{x}^{\infty} \mathrm{e}^{-\frac{y^{2}}{2}} d y
\end{array}
$$




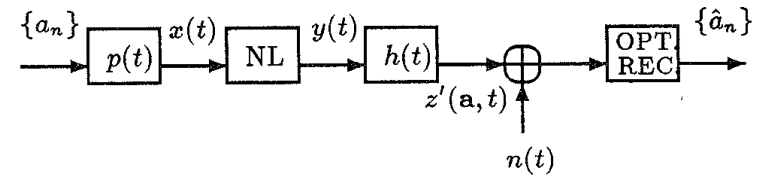

Fig. 1. Baseband equivalent of a nonlinear system with AWGN.

where $N_{0}$ is the one-sided power spectral density of $n(t)$ and

$$
d^{2}(\hat{\mathbf{a}}, \mathbf{a}) \triangleq \int_{T_{0}}\left|z^{\prime}(\hat{\mathbf{a}}, t)-z^{\prime}(\mathbf{a}, t)\right|^{2} d t
$$

is the Euclidean square distance between the two sequences (the integral extends over an interval $T_{0}$ including the entire transmission). Resorting to the classical concept of error event beginning at discrete time $n$, an error sequence $\mathbf{e}=$ $\left\{\ldots 0,0, e_{n}, e_{n+1}, \ldots, e_{n+D-L}, 0,0, \ldots\right\}$ can be associated to any such error event, where $D$ is the duration and $L$ is the channel memory. Thus, the probability that an error event starts at time $n, E \triangleq \bigcup\{\mathbf{e}\}$, can be expressed as

$$
\begin{aligned}
P\{E\} & =P\{\bigcup\{\mathbf{e}\}\}=\sum_{\mathbf{e}} P\{\mathbf{e}\} \\
& =\sum_{\mathbf{e}} \sum_{\mathbf{a} \in A(\mathbf{e})} P(\mathbf{a}+\mathbf{e} \mid \mathbf{a}) P\{\mathbf{a}\}
\end{aligned}
$$

where the set $A(\mathbf{e})$ contains all the transmitted sequences a, with a-priori probability $P\{\mathbf{a}\}$, for which $\hat{\mathbf{a}}=\mathbf{a}+\mathbf{e}$ is an allowable information sequence. Equations (3) and (5) can be combined to give a union bound on the error probability. For high signal to noise ratio, we can further simplify this bound by including only the dominant terms which involve the minimum distance

$$
d_{\min } \triangleq \min _{\mathbf{a}, \mathbf{e}} d(\mathbf{a}+\mathbf{e}, \mathbf{a})
$$

The simplified approximate bound then reads

$$
P\{E\} \widetilde{<} Q\left[\frac{d_{m i n}}{\sqrt{2 N_{0}}}\right] \sum_{\mathbf{e} \in E_{m i n}} P\{\mathbf{a} \in A(\mathbf{e})\}
$$

where $E_{\min }$ is the set of all possible error events with minimum distance starting at time $n$ (including all those error sequences e for which there exist some allowable sequences a which minimize, together with e, the above defined distance); $P\{\mathbf{a} \in A(\mathbf{e})\}$ is the probability that, for a given error sequence, the generic sequence $\mathbf{a}$ is such that $\mathbf{a}+\mathbf{e}$ is allowable.

The minimization of equation (6) is difficult since not only one must find a suitable expression for the received (noise-free) signal $z^{\prime}(\mathbf{a}, t)$, but also $d(\mathbf{a}+\mathbf{e}, \mathbf{a})$ depends on both the error sequence $\mathbf{e}$ and the transmitted sequence a. In the case of linear channels, the square distance is actually independent of the transmitted sequence and has expression

$$
\mathbf{s}^{2}(\mathbf{e}) \triangleq \int_{T_{0}}\left|z^{\prime}(\mathbf{e}, t)\right|^{2} d t
$$

which corresponds to the energy associated with the transmission of the error sequence $\left(z^{\prime}(\cdot)\right.$ has of course a different expression in the two cases).

Following this general approach, the minimum distance (6) could be numerically evaluated for given channel parameters [4]. In the following, we will introduce some simplifications and restrict the analysis to single-term sequences in order to point out the factors that influence the value of $d_{\min }$ as compared to the corresponding value obtained for $s_{\min }$ in the linear case.

\section{ERROR PERFORMANCE BOUNDS FOR CUBIC NONLINEARITIES}

\section{A. The distortion parameter $\xi$}

In this section, it will be assumed that the nonlinear transfer characteristic of the amplifying device is a third order real polynomial. In terms of the bandpass signals $u(t)$ and $v(t)$ at the input and output of the nonlinear device, respectively, we have

$$
v(t)=\gamma_{1} u(t)+\gamma_{3} u^{3}(t)
$$

where the coefficients $\gamma_{i}$ are assumed to be real. Under this assumption, it can be shown [6] that the nonlinear device has no AM/PM distortion and the AM/AM function relating the baseband equivalent of $u(t)$ and $v(t)$, denoted as $x(t)$ and $y(t)$ in fig. 1 , respectively, is such that equation (2) becomes

$$
y(t)=\gamma_{1} x(t)+\frac{3}{4} \gamma_{3} x(t)|x(t)|^{2}
$$

in which a second order term would not appear, even if it was present in (9), due to the bandpass nature of the nonlinearity.

Let us further assume that the modulation format is a multilevel PAM with symbols belonging to the normalized interval $[-1 ; 1]$ and the impulse response of the transmission filter is real, with maximum absolute amplitude $A_{M}$, and confined to a duration $T$ of one symbol. These assumptions are summarized in the following equations

$$
\begin{aligned}
& a_{n} \in[-1 ; 1] \Rightarrow e_{n} \in[-2 ; 2] \\
& |p(t)| \leq A_{M} \\
& p(t)=0 \text { if } \quad t \notin[0, T] .
\end{aligned}
$$

Under these assumptions, it is easy to see that the dynamic range of the baseband modulated PAM signal $x(t)$ that enters the amplifier is confined to the interval $\left[-A_{M}, A_{M}\right]$. If the characteristic (10) was perfectly linearized in order to obtain the same output level when the input takes on its peak level $A_{M}$, one would obtain a linear amplifier with gain $\alpha_{1}$, with $\alpha_{1}$ obeying the following equality

$$
\alpha_{1} A_{M}=\gamma_{1} A_{M}+\frac{3}{4} \gamma_{3} A_{M}^{3} .
$$




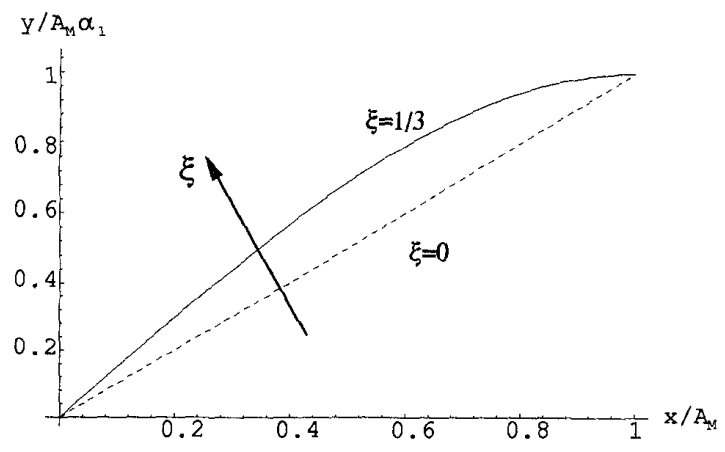

Fig. 2. Cubic AM/AM characteristics for various values of $\xi$.

The linear amplifier can be thought of as obtained from a variety of cubic curves, all reaching the same level $\alpha_{1} A_{M}$ when their input takes on the peak value $A_{M}$. It is useful to define a parameter

$$
\xi \triangleq \frac{\gamma_{1}-\alpha_{1}}{\gamma_{1}} \quad ; \quad 0 \leq \xi \leq \frac{1}{3},
$$

which will be referred to as the distortion factor, in order to express the coefficients $\gamma_{1}$ and $\gamma_{3}$ as a function of $\xi$ and $A_{M}$. From the last two equations we can rewrite $(10)$ as

$$
y(t)=\alpha_{1} \frac{1}{1-\xi} x(t)-\alpha_{1} \frac{\xi}{1-\xi} \frac{x^{3}(t)}{A_{M}^{2}}
$$

where the modulus $|\cdot|$ has been omitted since the signal $x(t)$ is real.

Fig. 2 shows, on normalized axes, the AM/AM transfer characteristics (16) of cubic nonlinear devices for positive values of the input signal $x$. Curves are plotted for the limiting values of the distortion parameter $\xi$. The dashed line shows the linear transfer characteristic, with unit gain in the normalized scale, obtainable through linearization of the device under a peak power constraint. It is easily seen from this figure that the distortion factor identifies cubic curves with increasing saturating effect as it goes from zero (the cubic degenerates into the dashedline) to $\frac{1}{3}$. We restrict our attention to values in the interval $\left[0, \frac{1}{3}\right]$, as stated in (15), so that all the curves reach the same peak value when $x$ takes on its maximum value $A_{M}$.

\section{B. Performance on wideband channels}

The signal $y(t)$ at the output of the cubic device has a broader spectrum than $x(t)$. If the physical channel $h(t)$ has flat frequency response in the bandwidth of $y(t)$, the corresponding block can be omitted from fig. 1 . In this case, the noiseless signal $z^{\prime}(\mathrm{a}, t)$ entering the receiver coincides with $y(t)$ and can be written as

$$
\begin{aligned}
z^{\prime}(\mathbf{a}, t) & =\alpha_{1} \frac{1}{1-\xi} \sum_{n} a_{n} p(t-n T) \\
& -\frac{3}{4} \frac{\alpha_{1}}{A_{M}^{2}} \frac{\xi}{1-\xi} \sum_{i} a_{i}^{3} p^{3}(t-i T)
\end{aligned}
$$

where the triple summation that would stem from the term $x^{3}(t)$ in (16) can be reduced to a single one using our assumption that transmission pulses associated with different symbols are nonoverlapping. Note that the assumption of finite duration for $p(t)$ would require an infinite bandwidth for the physical channel: in the numerical results, we will employ a large finite bandwidth, yielding an approximately finite duration for $p(t)$.

Equations (8) and (4) define, in the linearized and nonlinear case, the square distances to be minimized. By proper manipulation, the integrals in (8) and (4) can be "sliced" in the summation of nonoverlapping terms, each corresponding to a single transmitted symbol. Since the terms to be added are all non negative, the search for the minimum can be restricted to a single symbol period in which a symbol $a$ is transmitted and a symbol $a+e$ is detected by the receiver. Hence, the minimum square distance in the two cases is

$$
\begin{gathered}
s_{\min }^{2}=\min _{e} s^{2}(e)=\min _{e}\left\{\int_{0}^{T}\left|\alpha_{1} e p(t)\right|^{2} d t\right\} \\
d_{\min }^{2}=\min _{a, e} d^{2}(a+e, a)=\min _{a, e}\left\{\int_{0}^{T} \mid \alpha_{1} \frac{1}{1-\xi} e p(t)\right. \\
\left.-\left.\frac{3}{4} \frac{\alpha_{1}}{A_{M}^{2}} \frac{\xi}{1-\xi}\left(e^{3}+3 e^{2} a+3 e a^{2}\right) p^{3}(t)\right|^{2} d t\right\}=(19) \\
\min _{a, e}\left\{\int_{0}^{T}\left|\alpha_{1} e p(t)\right|^{2}\left|\frac{1-\xi \frac{p^{2}(t)}{A_{M}^{2}} \frac{3}{4}\left[e^{2}+3 e a+3 a^{2}\right]}{1-\xi}\right|^{2} d t\right\}
\end{gathered}
$$

The above reasoning is intuitively sound if one thinks that, because of the assumptions made, the signal observed by the optimal receiver is free of ISI and the receiver follows a symbol-by-symbol decision rule computing the distance between sequences as a sum of distances between corresponding symbols of these sequences. Since in our case the channel is memoryless, single symbol errors dominate the probability of error event.

The minimization in (18) is trivially performed by choosing $e_{m}=-\frac{2}{M-1}$, i.e., with minimum absolute value (recall the normalization (11) of the symbol set). This corresponds to considering symbol errors that occur between two adjacent symbols. In order to minimize (20), note that the first factor in the integrand is minimized for $e=e_{m}$ and the term in square brackets defines an elliptical paraboloid in the $(a, e)$ plane with a single absolute minimum, reaching its maximum on the border of the allowable domain defined by the relations

$$
\begin{aligned}
& a \in[-1,1] \\
& e \in[-1-a, 1-a]
\end{aligned}
$$

(which assure that both $a+e$ and $a$ belong to the symbol set). The minimum is reached in the symmetrical points $(a, e)=(1,0)$ and $(a, e)=(-1,0)$, with 


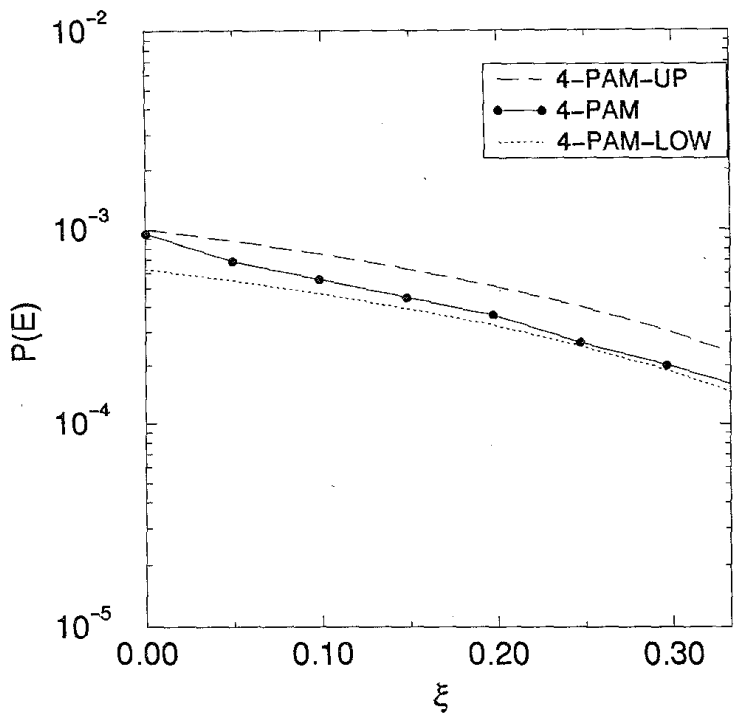

Fig. 3. Error performance for cubic channel (4-PAM).

an equal value of 3 . If $\xi \leq \frac{4}{9}$, these points are those which minimize the whole integrand and, thus, the square distance. Of course $e=0$ is not an allowable error symbol; hence, the couples minimizing the distance are $\left(a_{M}, e_{m}\right),\left(-a_{M},-e_{m}\right),\left(-a_{M}-e_{m}, e_{m}\right)$ and $\left(a_{M}+e_{m},-e_{m}\right)$ where $a_{M}=1$ is the symbol with maximum absolute value. These couples are obtained by considering only those errors that occur when the largest symbol is transmitted and its "closest" neighbouring symbol is estimated by the receiver or viceversa.

A comparison of the two expressions for $s_{\min }^{2}$ and $d_{\min }^{2}$ shows that the integrand of the latter includes two factors, the first being the integrand which defines $s_{\text {min }}^{2}$. Note that the condition

$$
\frac{p^{2}(t)}{A_{M}^{2}} \frac{3}{4}\left[e^{2}+3 e a+3 a^{2}\right]<1 \quad \forall t
$$

would cause the integrand in $d_{m i n}^{2}$ to be larger than the corresponding integrand in $s_{\min }^{2}$ for any $t$, resulting in an improved performance in the nonlinear channel as compared to the linearized one. Although condition $(22)$ is usually not verified, it intuitively suggests that transmission pulses $p(t)$ close to their peak value for their whole duration are penalizing for the nonlinear channel. As an example, if the term in square brackets in (22) is larger than $\frac{4}{3}$, for a rectangular pulse $p(t)=A_{M}$ of duration equal to the symbol interval, condition (22) is verified with a reversed sign ( $>$ ), implying $d_{\min }<s_{\min }$ and a better performance for the linearized channel. The statement above has been numerically verified through simulation for four different transmission pulses $p(t)$.

Rewriting (7) for the nonlinear channel yields the

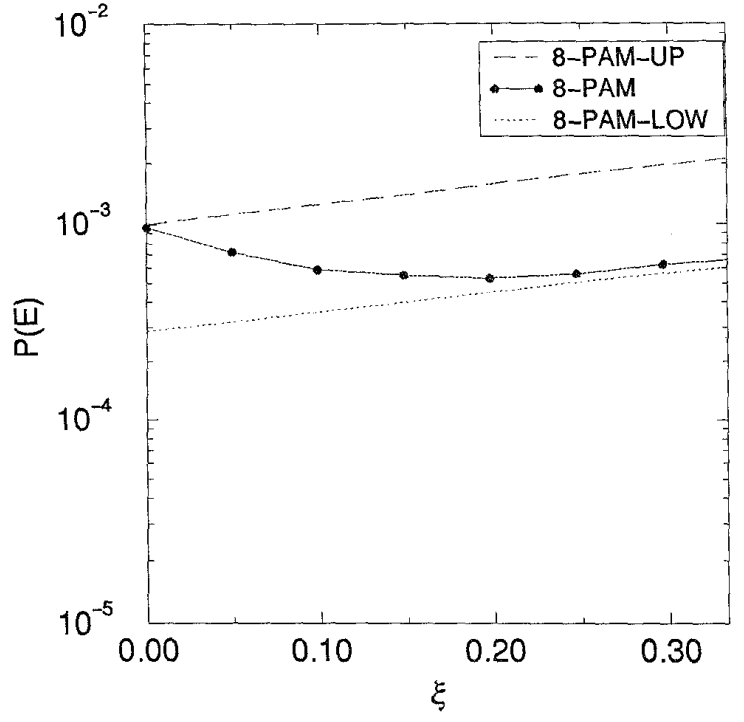

Fig. 4. Error performance for cubic channel (8-PAM).

following upper bound on the symbol error probability

$$
\begin{aligned}
P\{E\} & \widetilde{<} Q\left[\frac{d_{\min }}{\sqrt{2 N_{0}}}\right] \sum_{e \in E_{\min }} P\{a \in A(e)\} \\
& =2 \frac{M-1}{M} Q\left[\frac{d_{\min }}{\sqrt{2 N_{0}}}\right]
\end{aligned}
$$

and a similar bound, with $s_{\min }$ in place of $d_{\min }$, for the linearized channel; the set $E_{\text {min }}$ consists now of the error symbols $\pm e_{m}$. The above expression corresponds to assuming an equal pairwise error probability, with associated distance $d_{m i n}$, for any transmitted symbol $a$ for which $a+e$ is an allowable symbol; i.e. a uniform error property applies. Although this property holds true for the case of linear channels, it does not in the case of nonlinear channels.

We could further simplify the bound for the nonlinear channel by considering, for any $e \in E_{m i n}$, only those allowable symbols which minimize, together with $e$, the distance (20). In this case, there are only two symbols to be considered, instead of $(M-1)$, for each term of the summation in (23) ( $a=a_{M}$ or $a=-a_{M}-e_{m}$, for $e=+e_{m}$, and similarly for $\left.e=-e_{m}\right)$ and we obtain

$$
P\{E\}>\frac{4}{M} Q\left[\frac{d_{\min }}{\sqrt{2 N_{0}}}\right] .
$$

This is of course a lower bound since, referring to the summations in (5), all the terms have been disregarded except four. In other words, all possible errors associated with symbols other than the four symbols which minimize the distance, are assumed to have a zero probability of error. We can expect that when the distortion parameter $\xi$ approaches zero, i.e., the system is almost linear, the upper bound (23) is closer to 


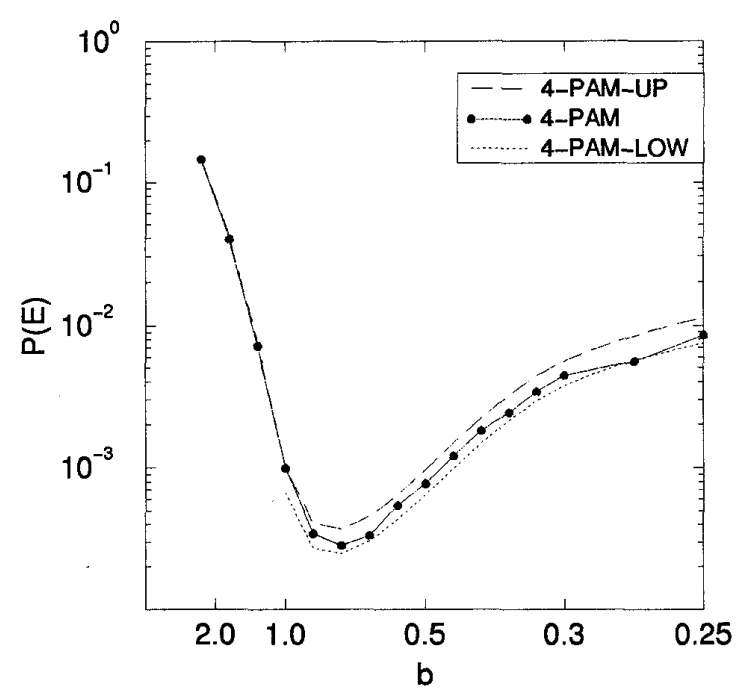

Fig. 5. Error performance for soft-limiting channel (4-PAM).

actual performance, whereas for more saturating devices the dominating errors occur only for the farthest symbols of the symbol set, bringing the actual performance close to the lower bound (24).

\section{SOFT-LIMITING NONLINEARITIES}

Cubic nonlinearities do not cause severe saturating effects, even for large values of the previously introduced distortion parameter. In order to evaluate the effects of sharper nonlinearities on error performance, in this section we will consider the same system model and assumptions of the previous section but we will replace the nonlinear device in fig. 1 with one having an input-output characteristic $F[\cdot]$ modeled as a softlimiter:

$$
F[x(t)]=\left\{\begin{array}{lll}
\alpha_{1} x(t) & \text { for } & |x(t)| \leq A_{M} \\
\alpha_{1} A_{M} & \text { for } & |x(t)|>A_{M}
\end{array}\right.
$$

In order to allow various degrees of nonlinear distortion for this type of nonlinear function, we introduce an Input Back-Off (IBO) factor, denoted as $b$, which may be considered as a linear attenuation applied to the signal $x(t)$ entering the nonlinearity. If $b=1$ there is no attenuation and, since $x(t)$ has a peak value of $A_{M}$, the amplifier is used only in its linear region; if $b<1, x(t)$ is amplified with various degrees of saturation, depending on $b$. The expression for $z^{\prime}(\mathbf{a}, t)$ in this case becomes

$$
z^{\prime}(\mathbf{a}, t)=\sum_{n} F\left[\frac{1}{b} a_{n} p(t-n T)\right]
$$

having used the assumption that transmission pulses associated with different symbols do not overlap. The optimal receiver estimates the transmitted sequence

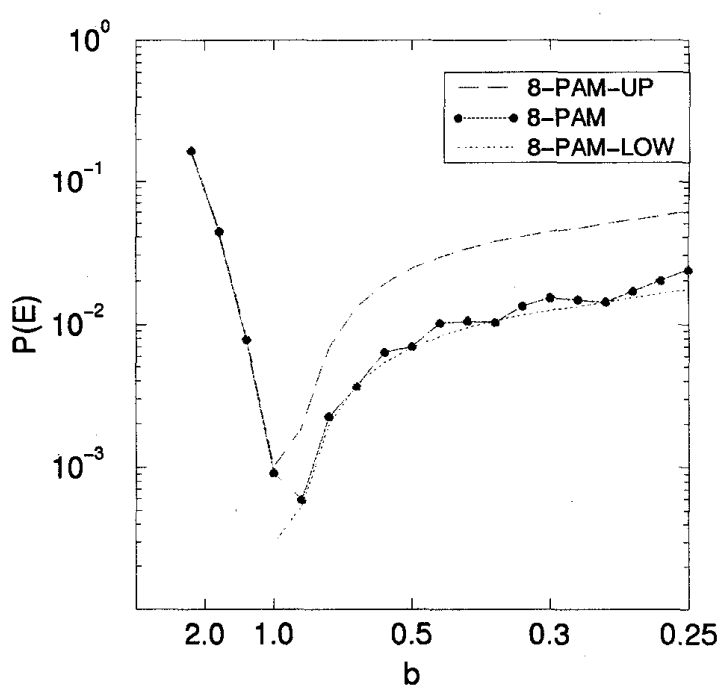

Fig. 6. Error performance for soft-limiting channel (8-PAM).

in a symbol-by-symbol fashion and the minimum distance can be again found between two sequences differing in one symbol only. This minimum distance may be expressed as follows

$d_{\text {min }}^{2}=\min _{a, e}\left\{\int_{0}^{T}\left|F\left[\frac{1}{b}(a+e) p(t)\right]-F\left[\frac{1}{b} a p(t)\right]\right|^{2} d t\right\}$

In this case, the minimization of the above expression is in general not analytically feasible, but it is intuitive that the minimum square distance is reached for $a=a_{M}$ and $e=e_{m}$. This can be proved since the derivative of the integral in (27), with respect to $e$, is zero for $e=0$ (for which $d_{\min }=0$ ) and the integral itself is continuous with $e$, whereas, for a fixed $e$, the integral is monotonically decreasing for increasing values of $|a|$.

Thus the same upper and lower bounds of equations (23) and (24) apply to the case of a soft-limiting nonlinearity, with $d_{\min }$ computed numerically.

\section{Numerical RESUlts}

Computer simulation has been performed in both the previously introduced nonlinear channels. In the simulated systems, pulse $p(t)$ has been chosen to have a strictly bandlimited transfer function, corresponding to the first lobe of a $\operatorname{sinc}^{2}\left(\frac{f}{B}\right)$ function. The bandwidth $B$ is chosen to be equal to $\frac{8}{3}$ times the signaling frequency so that, for a cubic nonlinearity, the two-side bandwidth of $y(t)$ is $\frac{16}{T}$; hence, $y(t)$ can be represented by 16 samples per symbol period. In both cases, cubic or soft-limiting nonlinearity, a channel filter $h(t)$ has been employed with an ideal low-pass transfer function with bandwidth equal to 16 times the signaling frequency; this introduces no linear distortion on $y(t)$ 
in the case of cubic devices. In the case of soft-limiting devices, $z^{\prime}(\mathbf{a}, t)$ is strictly bandlimited by $h(t)$, as necessary for simulation purposes; hence suboptimal performance must be expected with respect to the theoretical results.

The bounds in (23) and (24) have been numerically evaluated, with channel parameters chosen as outlined. Figures 3 and 4 show the previously derived upper and lower bounds along with the simulated Symbol Error Probability (SEP) versus the distortion parameter $\xi$, with $\xi$ varying between 0 and $\frac{1}{3}$, for a third order channel and a 4-PAM and 8-PAM symbol set, respectively. The noise power spectral density $N_{0}$ has been chosen to obtain a constant SEP of $10^{-3}$ on the linearized channel. In the case of 4-PAM, a constant improvement is observed for increasing distortion, with SEP improvements up to an order of magnitude. For 8PAM there is an optimal value for the distortion parameter $\xi$, but performance is roughly constant. Note how, for increasing values of the symbol set cardinality $M$, the two bounds tend to split apart; this is evident from the expressions (23) and (24) of the upper and lower bounds. The system is linear for $\xi=0$ : simulated performance and the upper bound coincide at this point, as expected.

Figures 5 and 6 show the previously derived upper and lower bounds, along with the simulated SEP versus the IBO parameter $b$, for a soft-limiting channel with the same signal sets and the same choice for $N_{0}$ as in the previous case. On the horizontal axis, the scale for $b$ is chosen so as to have a linearly increasing value for the gain $\frac{1}{b}$, from left to right. As expected, when $b \geq 1$ the device is employed in its linear region and the simulated performance has a typical $Q(\cdot)$ shape, perfectly overlapped with the upper bound (which is accurate since the uniform error property applies). The lower bounds were plotted only for $b \leq 1$ since they only apply when the amplifier is saturated. In the case of 8-PAM, there is a sudden performance degradation, however, the SEP remains within an order of magnitude $\left(10^{-2}\right)$ even for small values of $b$. In the 4-PAM case, there is an optimal value for $b$ corresponding to a small amount of distortion, whereas for $b=0.5$, half of the employed dynamic range of the amplifier is completely saturated and performance is comparable to that of the linearized channel $\left(10^{-3}\right)$.

In all the figures, simulated curves tend to approach the lower bounds, even for small amounts of saturation of the nonlinear device: this means that symbol errors tend to concentrate on the outmost symbols of the set when the system becomes nonlinear.

\section{Conclusions}

Two kinds of nonlinear ISI-free wideband channels have been considered, with amplifying devices having a cubic and soft-limiting nonlinear characteristic, respectively. A union bound on the symbol error probability has been recalled and considerations have been made for the cubic channel, which is more analytically tractable. Some factors affecting the performance have been identified, such as the impulse response of the transmission filter, the signal set cardinality and the degree of saturation of the nonlinearity. A lower bound including only those errors occurring on the 4 outmost symbols of the set has been introduced and its consistency verified through simulation.

Given a nonlinear channel of the considered types, for a "sparse" signal set such as 4-PAM, performance may be appreciably improved, with respect to that achievable through perfect linearization under a peak power constraint, by allowing nonlinear distortion, possibly selecting a proper back-off value. In fact, this causes an increased received mean-power which partially compensates for the receiver reduced capability to distinguish between saturated pulses associated with different symbols.

For signal sets with larger cardinality (8-PAM), performance degradation is observed when the nonlinearity is severely saturated, whereas for cubic devices with small saturating effects the nonlinear distortion may be completely recovered by an optimal receiver. Simulations and bounds have also been obtained for a 16PAM signal set, with results quantitatively similar to those of the 8-PAM case.

\section{REFERENCES}

[1] G. F. Hermann, "Performance of Maximum-Likelihood Receiver in the Nonlinear Satellite Channel", IEEE Trans. Commun., vol. COM-26, pp.373-378, March 1978.

[2] M. F. Mesiya, P. J. McLane and L. L. Campbell, "Maximum Likelihood Sequence Estimation of Binary Sequences Transmitted Over Bandlimited Nonlinear Channels", IEEE Trans. Commun., vol. COM-25, pp.633-643, July 1977.

[3] W. van Etten and F. van Vugt, "Maximum Likelihood Receivers for Data Sequences Transmitted over Nonlinear Channels", Archiv für Elektronik und Übertragungsteknik $(A E \ddot{U})$, vol.34, pp.216-223, 1980.

[4] A.Vannucci and R.Raheli "Optimal Sequence Detection Based on Oversampling for Bandlimited Nonlinear Channels", in Proc. IEEE Intern. Conf. Commun. (ICC '98), Atlanta (GA, U.S.A.), June 1998.

[5] S. Benedetto, E. Biglieri, V. Castellani, Digital Transmission Theory, Prentice-Hall, 1987.

[6] N. M. Blachman, "Detectors, Bandpass Nonlinearities, and Their Optimization: Inversion of the Chebyshev Trans form", IEEE Trans. Info. Theory, vol. IT-17, pp.398-404, July 1971. 